\title{
Microbubbles coupled to methotrexate-loaded liposomes for ultrasound-mediated delivery of methotrexate across the blood-brain barrier
}

This article was published in the following Dove Press journal:

International Journal of Nanomedicine

23 October 2014

Number of times this article has been viewed

\section{Xiang Wang' \\ Ping Liu' \\ Weixiao Yang' \\ $\mathrm{Lu} \mathrm{Li}^{\prime}$ \\ Peijing $\mathrm{Li}^{2}$ \\ Zheng Liu' \\ Zhongxiong Zhuo' \\ Yunhua Gao'}

'Department of Ultrasound, Xinqiao Hospital of the Third Military Medical University, Chongqing, ${ }^{2}$ Department of Ultrasound, General Hospital of the Jinan Military Area, Jinan, People's Republic of China
Correspondence: Yunhua Gao Department of Ultrasound, Xinqiao Hospital of the Third Military Medical University, Chongqing 400037,

People's Republic of China

Email gyh755631@।63.com

Weixiao Yang

Department of Ultrasound, Xinqiao Hospital of the Third Military Medical University, Chongqing 400037, People's Republic of China Email yangwx1973@I63.com

\begin{abstract}
Methotrexate (MTX) is the single most effective agent for the treatment of primary central nervous system lymphoma. Currently, the delivery of MTX to the brain is achieved by high systemic doses, which cause severe long-term neurotoxicity, or intrathecal administration, which is highly invasive and may lead to infections or hemorrhagic complications. Acoustically active microbubbles have been developed as drug carriers for the noninvasive and brain-targeted delivery of therapeutics. However, their application is limited by their low drug-loading capacity. To overcome this limitation, we prepared microbubbles coupled to MTX-loaded liposomes using ZHIFUXIAN, a novel type of microbubbles with a superior safety profile and long circulation time. MTX-liposome-coupled microbubbles had a high drug-loading capacity of $8.91 \% \pm 0.86 \%$, and their size $(2.64 \pm 0.93 \mu \mathrm{m}$ in diameter) was suitable for intravenous injection. When used with ultrasound, they showed more potent in vitro cytotoxicity against Walker-256 cancer cells than MTX alone or MTX-loaded liposomes. When Sprague-Dawley rats were exposed to sonication, administration of these MTX-liposome-coupled microbubbles via the tail vein led to targeted disruption of the blood-brain barrier without noticeable tissue or capillary damage. High-performance liquid chromatography analysis of the brain MTX concentration showed that MTX delivery to the brain followed the order of MTX-liposome-coupled microbubbles + ultrasound $(25.3 \pm 2.4 \mu \mathrm{g} / \mathrm{g})>$ unmodified ZHIFUXIAN + MTX + ultrasound $(18.6 \pm 2.2 \mu \mathrm{g} / \mathrm{g})>$ MTX alone $(6.97 \pm 0.75 \mu \mathrm{g} / \mathrm{g})>$ MTX-liposome-coupled microbubbles $(2.92 \pm 0.39 \mu \mathrm{g} / \mathrm{g})$. Therefore, treatment with MTX-liposome-coupled microbubbles and ultrasound resulted in a significantly higher brain MTX concentration than all other treatments $(P<0.01)$. These results suggest that MTX-liposome-coupled microbubbles may hold great promise as new and effective therapies for primary central nervous system lymphoma and other central nervous system malignancies.
\end{abstract}

Keywords: methotrexate, microbubbles, ultrasound, liposomes, blood-brain barrier

\section{Introduction}

Despite enormous advances in brain research, central nervous system (CNS) diseases remain the world's leading cause of disability. The main challenge of brain disease therapy is the blood-brain barrier (BBB). Endothelial tight junctions, together with an arsenal of enzymes, receptors, and specific transporters, prevent access of most drugs to the brain parenchyma. ${ }^{1}$ Many approaches have been explored to circumvent the BBB, both invasive and noninvasive, with their own promises and limitations. Drugs can be directly delivered to the brain via a needle or catheter inserted into the cranial cavity; ${ }^{2,3}$ however, this highly invasive method risks infection or brain trauma. Intranasal administration is a noninvasive option for drug delivery to the CNS. ${ }^{4}$ This method is emerging as a reliable method to bypass the BBB and deliver a wide range of 
therapeutic agents, including both small and large molecules, growth factors, viral vectors, and even stem cells to the brain and has shown therapeutic effects in both animals and humans. ${ }^{5}$ Nonetheless, there are some challenges that need to be addressed in intranasal delivery, especially of large molecular weight polar drugs such as peptides and proteins, including low membrane permeability, possibility of enzymatic degradation of the molecule in the lumen of the nasal cavity, and mucociliary clearance. ${ }^{6,7}$ Meanwhile, a number of drug modifications have been investigated to improve brain penetration. The addition of lipophilic groups increases drug delivery to the brain by passive diffusion. ${ }^{8}$ Drugs can also be also conjugated to targeting ligands, which activate receptor-mediated endocytosis of the drug-ligand complex, resulting in drug transport across the BBB. ${ }^{9}$ However, the delivery is often not brain-specific.

Agents temporarily disrupting the BBB have also been investigated. Intracarotid administration of hyperosmotic solutions like mannitol ${ }^{10}$ or vasodilators like bradykinin ${ }^{11}$ transiently increases BBB permeability and aids drug delivery to the CNS. These methods, however, are invasive procedures that require general anesthesia and can have side effects. ${ }^{12}$ Focused ultrasound (FUS) is an alternative noninvasive method to induce transient BBB disruption by elevating the temperature or creating gas bubbles in the targeted tissues. Previous studies have shown that FUS, although able to achieve BBB disruption, often results in tissue damage when used alone. ${ }^{13}$ In 2000, Hynynen et al found that if ultrasound is applied following intravenous injection of a microbubble contrast agent, the BBB could be opened without apparent brain tissue damage. ${ }^{14}$ Microbubbles are 1-10 $\mu \mathrm{m}$ gas-filled (with air or a perfluorocarbon) bubbles with a lipid or protein shell, and are commonly used as contrast agents for ultrasound imaging. During sonication, microbubbles expand and contract at the frequency of the ultrasound wave, resulting in BBB opening at a much lower acoustic power level. A variety of therapeutic agents including small molecules, antibodies, plasmid DNA, and siRNA, either alone or packaged in liposomes, have been successfully delivered across the BBB using FUS and microbubbles. ${ }^{15,16}$ This method is noninvasive and can achieve brain-specific delivery; thus, it has several advantages over other strategies to circumvent the BBB.

Microbubbles can be used as drug carriers as well. ${ }^{17-19}$ It is generally accepted that loading drugs directly into microbubbles provides greater control over drug release and deposition. However, since the microbubble shell is a thin lipid or protein membrane, its drug-loading capacity is quite limited. Efforts have been made to improve drug loading by incorporating phospholipids ${ }^{20}$ or surfactants ${ }^{21}$ into the shell, or coupling drug-loaded liposomes to the microbubble surface. ${ }^{18,22}$ The latter approach takes full advantage of the high drug-loading capacity of liposomes and the ultrasound-mediated BBB disruption effect of microbubbles. For example, Lentacker et al ${ }^{18}$ coupled doxorubicin-loaded liposomes to microbubbles via a biotin-avidin linkage. With ultrasound treatment, these doxorubicin-liposome-coupled microbubbles showed more rapid cellular uptake and more potent in vitro cytotoxicity than doxorubicin-loaded liposomes. However, whether these liposome-coupled microbubbles can be used for effective drug delivery across the BBB in vivo is not clear.

ZHIFUXIAN is a novel type of lipid perfluorocarbon microbubbles prepared in our laboratory. It is safe, stable, and demonstrates superior performance as a contrast agent for diagnostic ultrasound imaging. ${ }^{23,24}$ Additionally, our previous studies have shown that ZHIFUXIAN enhances ultrasoundmediated cisplatin transport across the BBB in an in vitro model. ${ }^{25}$ In the present study, we coupled methotrexate (MTX)-loaded liposomes to ZHIFUXIAN via a biotinavidin linkage and determined the physical and drug-loading properties of the conjugated microbubbles. We subsequently investigated the ultrasound-triggered biological effects of these MTX-liposome-coupled microbubbles on cancer cell proliferation and apoptosis in vitro, and BBB opening and brain MTX delivery in vivo.

\section{Materials and methods Preparation of MTX-loaded biotinylated liposomes}

Biotinylated liposomes were prepared following previously described procedures, ${ }^{23}$ with minor modifications. Briefly, biotin-1,2-distearoyl-sn-glycero-3-phosphoethanolamine (DSPE)-polyethylene glycol (PEG)2000, dipalmitoyl phosphatidyl-glycerol (DPPG), distearoyl phosphatidylcholine (DSPC), and PEG4000 in a mass ratio of $1: 1: 1: 150$ were mixed in ultrapure water to generate a liposome suspension. Meanwhile, 20\% MTX solution (w/w) was prepared by dissolving MTX (the same weight as that of the total lipids) in 1 $\mathrm{M} \mathrm{NaOH}$. Next, the liposome suspension and MTX solution prepared as above were thoroughly mixed on a vortex mixer, transferred to $3 \mathrm{~mL}$ vials $(1.5 \mathrm{~mL} / \mathrm{vial})$, and lyophilized following a temperature program of $-20^{\circ} \mathrm{C}$ for 6 hours, $-10^{\circ} \mathrm{C}$ for 6 hours, $0^{\circ} \mathrm{C}$ for 6 hours, and $20^{\circ} \mathrm{C}$ for 6 hours. The freezedried powder was subsequently hydrated with ultrapure water ( $1 \mathrm{~mL} / \mathrm{vial})$, oscillated on a mechanical shaker for 60 seconds, and lyophilized again as described above. The crude MTX-loaded, biotinylated liposomes were rehydrated with 
ultrapure water $(0.7 \mathrm{~mL} / \mathrm{vial})$ and filtered through a filter paper. The free MTX was removed by gel filtration on a Sephadex G-25 column $(60 \mathrm{~cm} \times 1.6 \mathrm{~cm})$. The particle size of the purified liposomes was analyzed by dynamic light scattering on a Zetasizer 3000 particle analyzer (Malvern Instruments, Malvern, UK). For morphological analysis, the liposomes were stained with $0.1 \%$ phosphotungstic acid for 10 minutes, washed with ultrapure water, and subjected to transmission electron microscopy (TEM; TECNAI-10, Philips, Eindhoven, the Netherlands). Finally, the purified, MTX-loaded biotinylated liposomes were transferred to $3 \mathrm{~mL}$ vials (1 mL/vial) and lyophilized as above.

\section{Preparation of biotinylated microbubbles}

Biotinylated ZHIFUXIAN microbubbles were prepared following previously described procedures, ${ }^{23}$ with minor modifications. Briefly, biotin-DSPE-PEG2000, DPPG, DSPC, and PEG4000 in a mass ratio of 1:1:1:150 were thoroughly mixed in ultrapure water by agitation at $60^{\circ} \mathrm{C}$. The lipid suspension was subsequently lyophilized. The dried powder was dispersed in $1 \mathrm{~mL}$ of a liquid mixture of $50 \%$ glucose, propylene glycol, and glycerin in a volume ratio of $8: 1: 1$. Next, the air in the vial was replaced with perfluoropropane. The vial was tightly sealed and oscillated on a mechanical shaker (AT \& M, Beijing, People's Republic of China) at a frequency of 4,000-5,500 per minute for 60 seconds to generate crude microbubbles. The crude microbubbles were thoroughly mixed with $10 \mathrm{~mL}$ of phosphate-buffered saline $(10 \mathrm{mM})$ and centrifuged at 1,000 rpm for 10 minutes. The lower layer was discarded, and the upper layer was washed three additional times with phosphate-buffered saline. Finally, the microbubbles were resuspended in approximately $2 \mathrm{~mL}$ of phosphate-buffered saline. To allow the coupling of biotinylated liposomes, biotinylated microbubbles were incubated with avidin ( $0.025 \mathrm{mg}$ of avidin per $\mathrm{mL}$ of microbubbles) at room temperature with shaking (60-70 rpm) for 15 minutes, washed, and resuspended in phosphate-buffered saline as described above.

\section{Coupling of MTX-loaded biotinylated liposomes to avidinylated microbubbles}

The MTX-loaded biotinylated liposomes (one vial) were mixed with avidinylated microbubbles $(\sim 2 \mathrm{~mL})$ and incubated at room temperature for 10 minutes. The liposomecoupled microbubbles were washed with phosphate-buffered saline by centrifugation as described above and stored at $-20^{\circ} \mathrm{C}$ for subsequent experiments. The morphology of the free and liposome-coupled microbubbles was studied by light microscopy, and their size distribution was determined on a Coulter particle analyzer (Omec Technology Co Ltd, Guangdong, People's Republic of China).

To visualize the attachment of liposomes to the microbubbles, daunomycin-liposome-coupled microbubbles were prepared using the procedures described above. The attachment of daunomycin-loaded liposomes to the microbubbles was visualized on a confocal laser microscope (Zeiss LSM510, Oberkochen, Germany) by detecting the orange-red fluorescence emission of daunomycin. The coupling efficiency was determined by analyzing the daunomycin-liposomecoupled microbubbles and the avidinylated microbubbles on a FACSCalibur flow cytometer (Becton Dickinson, Franklin Lakes, NJ, USA).

\section{Determination of drug loading and encapsulation efficiency}

The MTX-loaded liposomes and MTX-liposome-coupled microbubbles were ruptured by dissolution in methanol $(1 \mathrm{~mL} /$ vial for liposomes; $500 \mu \mathrm{L}$ for microbubbles). After methanol was removed by freeze-drying, the samples were rehydrated and subjected to high-performance liquid chromatography (HPLC) analysis on a C18 column. The MTX content in the samples was determined by measuring the absorbance of MTX at $306 \mathrm{~nm}$. The encapsulation efficiency (EE) and drug loading (DL) were calculated using Equations (1) and (2):

$$
\begin{gathered}
\mathrm{EE} \%=\mathrm{W}_{\text {encapsulated }} / \mathrm{W}_{\text {total }} \times 100 \% \\
\mathrm{DL} \%=\mathrm{W}_{\text {encapsulated }} / \mathrm{W}_{\text {particles }} \times 100 \%
\end{gathered}
$$

where $\mathrm{W}_{\text {encapsulated }}$ is the analyzed weight of MTX encapsulated in the particles (mg), $\mathrm{W}_{\text {total }}$ is the weight of MTX used to prepare the particles $(\mathrm{mg})$, and $\mathrm{W}_{\text {particles }}$ is the total weight of the freeze-dried particles.

\section{Cell culture}

Walker-256 tumor-bearing Sprague-Dawley rats were purchased from the Animal Center of Wuhan University. Rat ascitic fluid $(5 \mathrm{~mL})$ was collected and centrifuged at $1,000 \mathrm{rpm}$ for 5 minutes. The supernatant was removed, and the Walker-256 cancer cells were collected and washed twice with $10 \mathrm{mM}$ phosphate-buffered saline. Next, the cells were placed in Roswell Park Memorial Institute-1640 medium $\left(1 \times 10^{\%} / \mathrm{mL}\right)$ and cultured at $37^{\circ} \mathrm{C}$ with $5 \% \mathrm{CO}_{2}$ in a humidified incubator. After 24 hours, the medium was changed. Cells were subsequently cultured with a medium change every 2 days and passaged. Cells after passage 4 were used in subsequent studies. 


\section{In vitro cytotoxicity measurement}

Walker-256 cancer cells were seeded at $2 \times 10^{6} / \mathrm{mL}$ in six-well plates $(4 \mathrm{~mL} /$ well $)$ and cultured at $37^{\circ} \mathrm{C}$ and $5 \% \mathrm{CO}_{2}$ for 24 hours. The cells were subsequently divided into eight treatment groups as follows: control; MTX; MTX-loaded liposomes (MNL); MTX-liposome-coupled microbubbles (MNL-CA); ultrasound and MTX (US + MTX); ultrasound and MTX-loaded liposomes (US + MNL); ultrasound and MTX-liposome-coupled microbubbles (US + MNL-CA); and ultrasound and ZHIFUXIAN (US + UCA). MTX was added at a final concentration of $0.2 \mu \mathrm{M}$. MTX-loaded liposomes and MTX-liposome-coupled microbubbles were added at doses that contained the equivalent amount of MTX $(0.2 \mu \mathrm{M})$. In all experiments with ultrasound, ultrasound was applied using a Metron Accusonic Plus AP170 sonicator (Metron Medical, Carrum Downs, Australia) at the following settings: $1 \mathrm{MHz}$, $10 \%$ duty cycle, and an ultrasound intensity of $1 \mathrm{~W} / \mathrm{cm}^{2}$. Cells were immediately exposed to ultrasound for 10 seconds after addition of the treatment agents. After 24 hours, the medium was removed and the cells were incubated with $5 \mathrm{mg} / \mathrm{mL}$ 3-(4,5-dimethylthiazol-2-yl)-2,5-diphenyltetrazolium bromide (MTT) for 4 hours. Subsequently, dimethyl sulfoxide $(200 \mu \mathrm{L})$ was added and the cells were incubated with shaking for 10 minutes. Absorbance at $490 \mathrm{~nm}$ was measured to determine the amount of formed formazan. Cytotoxicity was calculated as percent inhibition of cell viability using Equation (3):

$$
\text { Inhibition }(\%)=\left[\left(\mathrm{OD}_{490, \mathrm{C}}-\mathrm{OD}_{490, \mathrm{~T}}\right) / \mathrm{OD}_{490, \mathrm{C}}\right] \times 100 \%
$$

where $\mathrm{OD}_{490, \mathrm{C}}$ is the absorbance of cells in the control group and $\mathrm{OD}_{490, \mathrm{~T}}$ is the absorbance of cells in the specific treatment groups.

\section{Cell apoptosis measurement}

After Walker-256 cancer cells were treated for 24 hours as above, they were collected and stained with propidium iodide and Annexin V using an Annexin V-FITC apoptosis detection kit (Abcam, Cambridge, MA, USA), according to the manufacturer's instructions. The cells were subsequently subjected to analysis on a FACSCalibur flow cytometer to determine cell apoptosis.

\section{Animal preparation}

Healthy Sprague-Dawley rats were purchased from the Laboratory Animal Center of Xinqiao Hospital affiliated with the Third Military Medical University. All experimental procedures were approved by the Animal Care and Use Committee at the hospital. All rats were anesthetized by intraperitoneal injection of pentobarbital $(30 \mathrm{mg} / \mathrm{kg})$. For intracranial ultrasound treatment, the fur in the area from the animal's ears to the top of the head was shaved using an electric razor. The remaining fur was removed using $8 \%$ sodium sulfide. Afterward, the scalp was rinsed thoroughly with tap water.

\section{Evaluation of BBB opening in rats using Evans blue}

One hundred rats with a mean weight of $240 \pm 34 \mathrm{~g}$ (range, 200-300 g) were prepared as described above and randomly divided into ten groups $(\mathrm{n}=10)$. Rats in the first nine groups were subjected to ultrasound treatment under various settings (Table 1) using a Metron Accusonic Plus AP170 sonicator. The probe of the sonicator was placed on top of the animal's head to deliver sonication to the brain. Immediately after the sonication started, MNL-CA were injected at various doses (Table 1) into the tail vein, followed by injection of $2 \%$ Evans blue (EB) $50 \mathrm{mg} / \mathrm{kg}$. Rats in the control group received EB injection only (group 10). At 2 hours after the EB injection, heparinized saline was perfused via the left ventricle until clear fluid oozed from the right atrium. The rats were then sacrificed, and the brains were isolated. After the olfactory bulb and cerebellum were removed, the brain was sectioned into small slices and extracted with formamide at $37^{\circ} \mathrm{C}$ for

Table I Treatment conditions for studying BBB disruption

\begin{tabular}{|c|c|c|c|c|}
\hline Group & $\begin{array}{l}\text { Ultrasound } \\
\text { intensity }\left(\mathrm{W} / \mathrm{cm}^{2}\right)\end{array}$ & $\begin{array}{l}\text { Duration } \\
\text { (minutes) }\end{array}$ & $\begin{array}{l}\text { Duty } \\
\text { cycle (\%) }\end{array}$ & $\begin{array}{l}\text { MTX-liposome-coupled } \\
\text { microbubbles (mL/kg) }\end{array}$ \\
\hline I & 1.0 & 2 & 10 & 0.2 \\
\hline 2 & 1.0 & 5 & 20 & 0.5 \\
\hline 3 & 1.0 & 10 & 50 & 1.0 \\
\hline 4 & 2.0 & 2 & 10 & 1.0 \\
\hline 5 & 2.0 & 5 & 20 & 0.5 \\
\hline 6 & 2.0 & 10 & 50 & 0.2 \\
\hline 7 & 2.4 & 2 & 50 & 0.5 \\
\hline 8 & 2.4 & 5 & 10 & 1.0 \\
\hline 9 & 2.4 & 10 & 20 & 0.2 \\
\hline
\end{tabular}

Abbreviations: BBB, blood-brain barrier; MTX, methotrexate. 
24 hours. Subsequently, the brain extracts were centrifuged at 3,000 rpm for 5 minutes, and the supernatants were subjected to analysis on a DU800 spectrophotometer (Beckman Coulter, Brea, CA, USA). The EB content in the brain was determined by measuring its absorbance at $620 \mathrm{~nm}$.

\section{Evaluation of MTX brain delivery in rats}

Forty rats with a mean weight of 260 $21 \mathrm{~g}$ (range, 250-280 g) prepared as described above were randomly divided into four groups $(\mathrm{n}=10)$ and treated as follows: US + MNL-CA; US + UCA + MTX; MNL-CA; and MTX. Brain ultrasound treatment was conducted as described above under the following settings: $1 \mathrm{MHz}, 20 \%$ duty cycle, and an ultrasound intensity of $2 \mathrm{~W} / \mathrm{cm}^{2}$. After MNL-CA $(0.5 \mathrm{~mL} / \mathrm{kg})$, ZHIFUXIAN $(0.5 \mathrm{~mL} / \mathrm{kg})$, or vehicle was injected into the tail vein, the rats were subjected to ultrasound treatment for 5 minutes. In the meantime, MTX $(2.5 \mathrm{mg} / \mathrm{kg}$, equivalent to the amount of MTX encapsulated in MNL-CA) or vehicle was injected into the tail vein, followed by injection of $0.5 \mathrm{~mL}$ of saline. Approximately 10 minutes later, heparinized saline was perfused via the left ventricle until clear fluid oozed from the right atrium. The rats were then sacrificed, and the brains were isolated. Approximately $100 \mathrm{mg}$ of brain tissue was collected from the parietal lobe region of the brain, wrapped in aluminum foil, and stored at $-70^{\circ} \mathrm{C}$ until analysis.

To determine the MTX content, the brain tissues were thawed, weighed, and homogenized in ice-cold water using an F8-6G homogenizer (Fluko, Essen, Germany). The brain tissue homogenates $(0.2 \mathrm{~mL})$ were thoroughly mixed with $0.2 \mathrm{~mL}$ of perchloric acid on a vortex mixer and centrifuged at 10,000 rpm for 5 minutes. The MTX content in the supernatants was determined by HPLC analysis. An MTX standard curve was established using MTX standards prepared in brain tissue homogenates from untreated rats.

\section{Histological analysis}

Twelve rats with a mean weight of $270 \pm 24 \mathrm{~g}$ (range, 250-300 g) prepared as described above were randomly divided into three groups ( $\mathrm{n}=4)$ and treated as follows: control (no treatment); US; and US + MNL-CA. The ultrasound settings used were as follows: $1 \mathrm{MHz}, 20 \%$ duty cycle, ultrasound intensity of $2 \mathrm{~W} / \mathrm{cm}^{2}$, and duration of 5 minutes. After ultrasound treatment was completed, the rats were perfused with heparinized saline as above. The rats in each group were then further divided into two subgroups $(n=2)$. Rats in the first subgroup were perfused via the left ventricle with $10 \%$ buffered neutral formalin for 20 minutes. The brains were immediately removed, and the region at the focal site of sonication was isolated and fixed in $10 \%$ buffered neutral formalin. The fixed tissues were then embedded in paraffin and sectioned. Every tenth section was stained with hematoxylin-eosin and examined microscopically for tissue and capillary damage. In the meantime, rats in the second subgroup were perfused via the left ventricle with cacodylate buffer containing lanthanum nitrate as a tracer for 20 minutes. Afterward, the rats were stored at $4^{\circ} \mathrm{C}$ overnight. The next day, the brains were isolated and tissue $\left(\sim 1 \mathrm{~mm}^{3}\right)$ was collected from the cerebral cortex and hippocampus region. The tissues were fixed in cacodylate buffer without lanthanum nitrate for 1 hour, washed, and dehydrated in ethanol. The tissues were then embedded in paraffin, sectioned, and subjected to TEM analysis.

\section{Statistical analysis}

All data were analyzed using Statistical Package for the Social Sciences version 13.0 software (SPSS Inc, Chicago, IL, USA). Results are presented as the mean \pm standard deviation. Statistical differences in the in vitro studies were analyzed by one-way analysis of variance. Statistical differences in the in vivo studies were analyzed by one-way analysis of variance or the Student's $t$-test.

\section{Results \\ Characterization of MTX-loaded liposomes and MTX-liposome-coupled microbubbles}

First, we prepared MTX-loaded biotinylated liposomes composed of biotin-DSPE-PEG2000, DPPG, DSPC, and PEG4000 in a mass ratio of 1:1:1:150. After MTX loading and purification by gel filtration, liposomes with diameters in the range of 50-200 nm were obtained, which had a spherical morphology as revealed by TEM (Figure 1). HPLC analysis showed that these

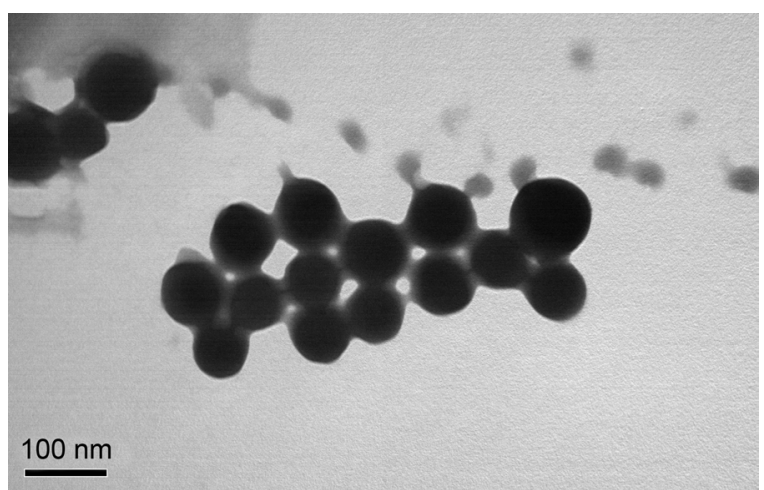

Figure I Transmission electron microscopic image of methotrexate-loaded biotinylated liposomes, composed of biotin-DSPE-PEG2000, dipalmitoyl phosphatidyl-glycerol, distearoyl phosphatidylcholine, and PEG4000, showing spherical morphology. Note: Bar $100 \mathrm{~nm}$.

Abbreviations: DSPE, I,2-distearoyl-sn-glycero-3-phosphoethanolamine; PEG, polyethylene glycol. 

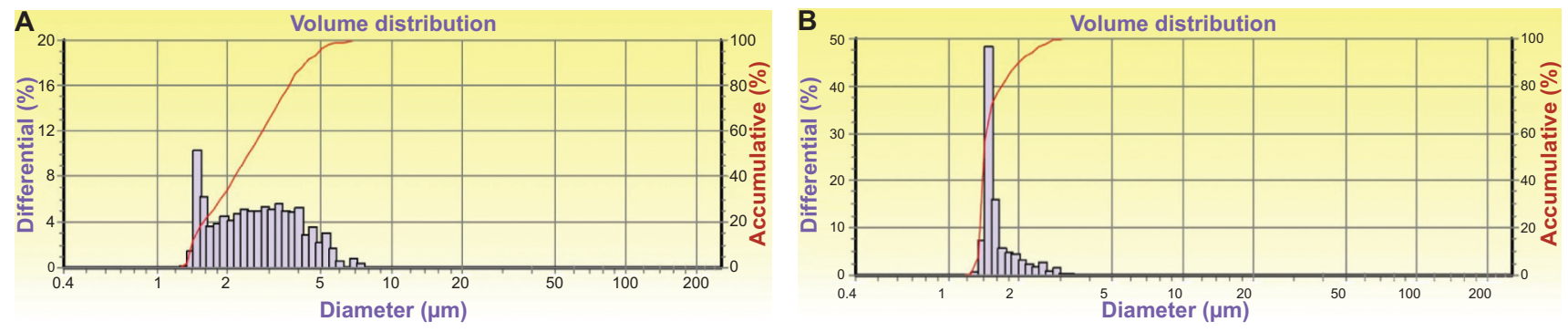

Figure 2 Size distribution of methotrexate-liposome-coupled microbubbles.

Notes: The size distribution of methotrexate-liposome-coupled microbubbles (A) was different from that of unmodified ZHIFUXIAN microbubbles (B).

particles had an EE of $83.4 \% \pm 4.5 \%$ and a DL of $31.4 \% \pm 2.5 \%$. Next, we prepared biotinylated ZHIFUXIAN microbubbles containing biotin-DSPE-PEG2000, DPPG, DSPC, or PEG4000 in a mass ratio of 1:1:1:150. These microbubbles were avidinylated and then coupled to biotinylated MTXloaded liposomes. The MTX-liposome-coupled microbubbles obtained had an average diameter of $2.64 \pm 0.93 \mu \mathrm{m}$, which was slightly larger than that of the unmodified ZHIFUXIAN microbubbles $(1.53 \pm 0.80 \mu \mathrm{m})$. In addition, their size distribution was different from that of ZHIFUXIAN (Figure 2), albeit in a range suitable for intravenous administration. Drug content analysis by HPLC showed that they had a DL of $8.91 \% \pm 0.86 \%$. Specifically, the conjugation between the liposomes and microbubbles was highly efficient in this study. Our flow cytometry analysis showed that $94 \%$ of the avidinylated microbubbles were successfully coupled to the daunomycin-loaded biotinylated liposomes. Furthermore, confocal fluorescence microscopy of the daunomycinliposome-coupled microbubbles revealed that the liposomes were evenly attached to the microbubble surface.

\section{In vitro cytotoxicity}

After we characterized the physical and morphological properties of the MTX-liposome-coupled microbubbles, we studied their effects on the proliferation of Walker-256 cancer cells with or without sonication using the MTT assay. After incubation without ultrasound treatment for 24 hours, MTXliposome-coupled microbubbles reduced the cell viability by $25.0 \% \pm 2.2 \%$, while MTX and MTX-loaded liposomes showed a higher cell killing effect with $50.1 \% \pm 5.0 \%$ and $62.5 \% \pm 6.4 \%$ inhibition, respectively (Figure 3 ). However, sonication greatly enhanced the cell killing effect of MTXliposome-coupled microbubbles, while having little effect on that of MTX or MTX-loaded liposomes. Consequently, MTX-liposome-coupled microbubbles became significantly more toxic to the cells than MTX or MTX-loaded liposomes (Figure 3). These effects of sonication were probably mediated by the increased release of MTX-loaded liposomes and free MTX from the microbubbles, and their accelerated cellular uptake following sonication-triggered cell membrane permeation. Unmodified ZHIFUXIAN with sonication (US + UCA) showed a cell killing effect of only $14.6 \% \pm 1.7 \%$ inhibition, the lowest among all the treatment groups, meaning that microbubbles with sonication were not causing a toxic effect on cells per se. Similar results were obtained from our cell apoptosis study using flow cytometry. When used without sonication, MTX-liposome-coupled microbubbles were less potent than MTX and MTX-loaded liposomes in inducing

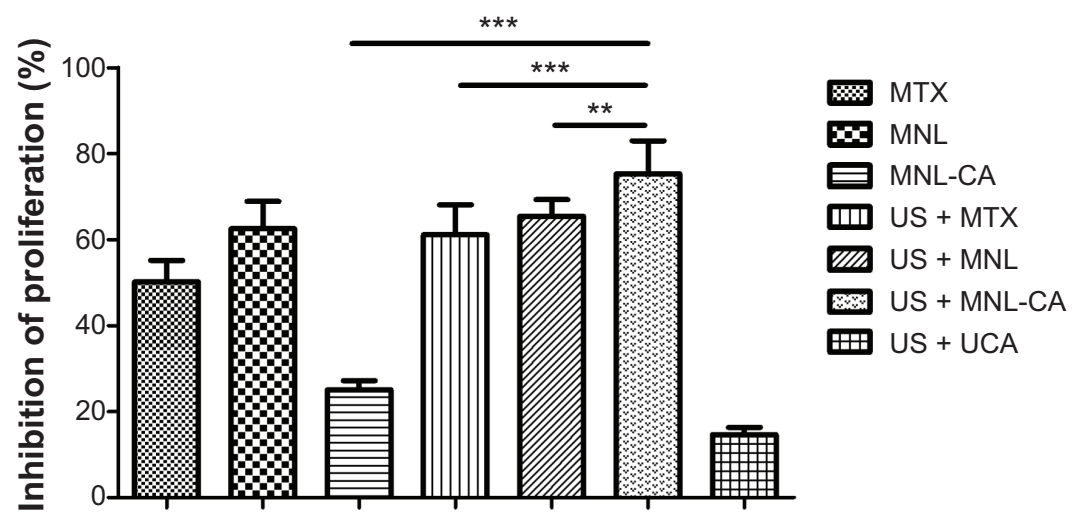

Figure 3 Inhibition of proliferation of Walker-256 cells after treatment for 24 hours with or without sonication. Notes: Data are presented as the mean \pm standard deviation $(n=9)$. $* * P<0.01$; $* * * P<0.001$.

Abbreviations: MTX, methotrexate; MNL, MTX-loaded liposomes; MNL-CA, MTX-liposome-coupled microbubbles; US, ultrasound; UCA, unmodified ZHIFUXIAN. 


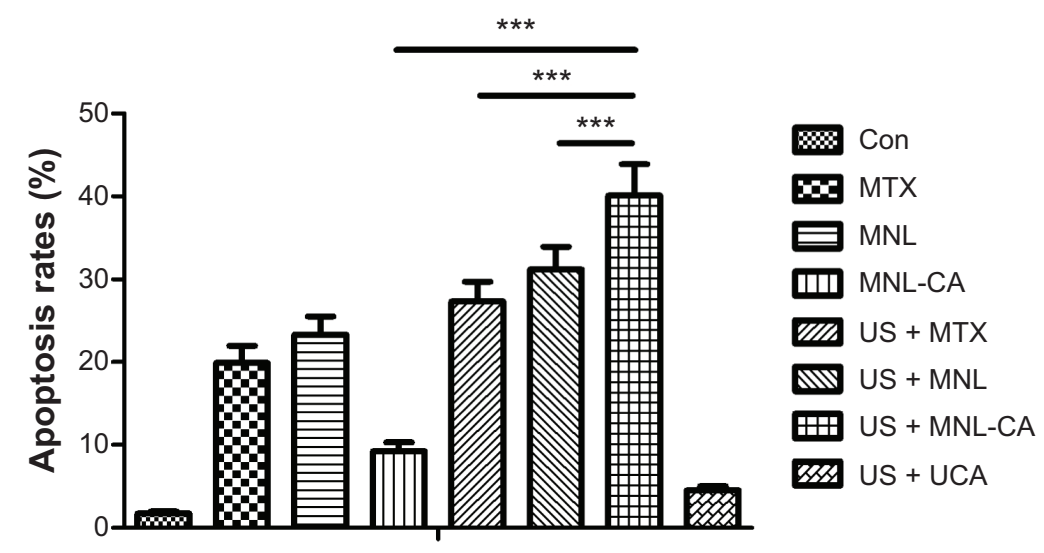

Figure 4 Induction of apoptosis in Walker-256 cells after treatment for 24 hours with or without sonication. Notes: Data are presented as the mean \pm standard deviation $(n=9)$. $* * * P<0.001$.

Abbreviations: Con, control; MTX, methotrexate; MNL, MTX-loaded liposomes; MNL-CA, MTX-liposome-coupled microbubbles; US, ultrasound; UCA, unmodified ZHIFUXIAN.

apoptosis of Walker-256 cells, but became significantly more potent after application of ultrasound (Figure 4).

\section{$\mathrm{BBB}$ opening in rats evaluated by $\mathrm{EB}$ extravasation}

$\mathrm{EB}$, an azo dye with very high affinity for albumin, is commonly used to assess the integrity of the BBB. Because serum albumin cannot cross the barrier, albumin-bound EB is constrained within the blood vessels when the BBB is intact, but enters the $\mathrm{CNS}$ if the $\mathrm{BBB}$ has been compromised. In this study, we used EB to monitor the extent of BBB disruption in rats following treatment with MTX-liposome-coupled microbubbles and ultrasound under the conditions shown in Table 1.

No visible EB staining was noted in the brains of control rats, which received EB injection only (Figure 5A). Varying degrees of brain EB staining were observed in groups 3-9 (Figure 5B), which were correlated with the amount of $\mathrm{EB}$ detected in the brain extracts (Figure 6). While permeability of the BBB markedly increased in groups $7-9$, vascular damage was also noted; therefore, these conditions were too harsh for the animals. The rats in groups 5 and 6 displayed significantly increased EB brain extravasation, with no noticeable tissue or capillary damage. Since the dosage of MTX-liposome-coupled microbubbles used in group $5(0.5 \mathrm{~mL} / \mathrm{kg})$ was higher than that used in group $6(0.2 \mathrm{~mL} / \mathrm{kg})$, the delivery of MTX across the BBB would probably be more effective under the conditions used in group 5. Therefore, we chose to use the conditions of group 5 (ultrasound intensity of $2 \mathrm{~W} / \mathrm{cm}^{2}$, duration of 5 minutes, duty cycle of $20 \%$, and $0.5 \mathrm{~mL} / \mathrm{kg}$ MTX-liposomecoupled microbubbles) for subsequent experiments.

\section{MTX delivery across the BBB in rats}

After we identified the optimal conditions for effective BBB disruption using MTX-liposome-coupled microbubbles and ultrasound, we investigated the delivery of MTX across the BBB by determining the concentration of MTX in the parietal lobe region of the brain. When MTX was administered without sonication, $6.97 \pm 0.75 \mu \mathrm{g}$ of MTX per gram of tissue was detected in the parietal lobe after treatment (Figure 7). When MTX-liposome-coupled microbubbles were administered without sonication, only $2.92 \pm 0.39 \mu \mathrm{g} / \mathrm{g}$ of MTX was detected in the above region. However, if
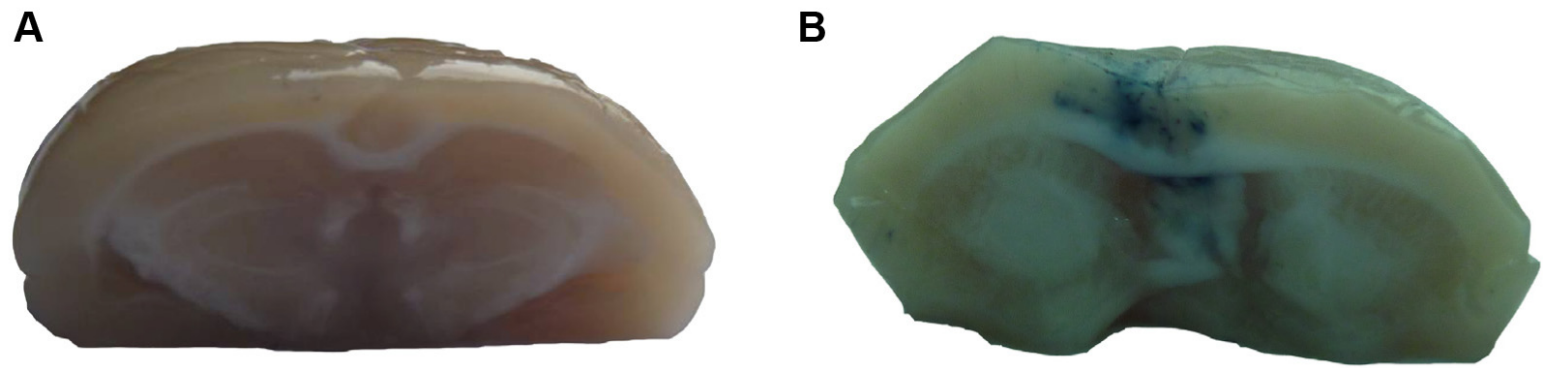

Figure 5 Microphotographs of Evans blue staining in rat brains to monitor the extent of blood-brain barrier disruption in rats following treatment with methotrexateliposome-coupled microbubbles and ultrasound.

Notes: (A) Rats receiving Evans blue injection only. (B) Rats treated with methotrexate-methotrexate-liposome-coupled microbubbles and ultrasound under the conditions shown for group 5 in Table 1 . 


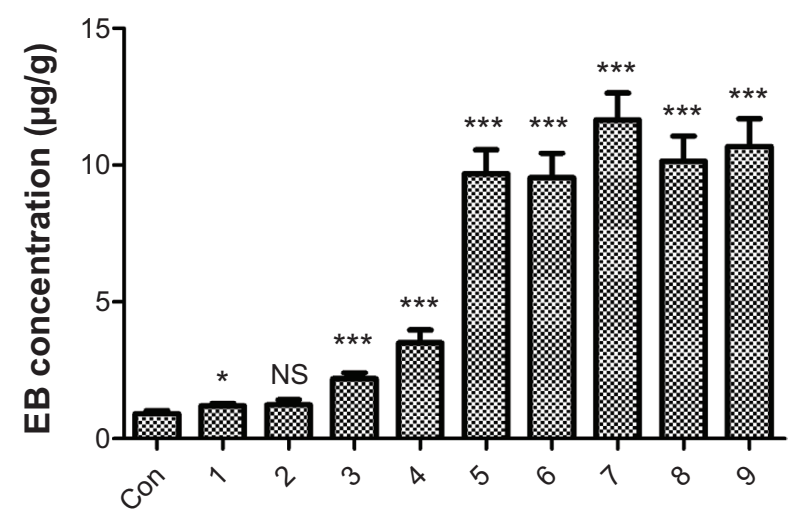

Figure 6 EB extravasation into the rat brain following the treatments shown in Table I.

Notes: EB was extracted from the brain tissues and determined by absorbance at $620 \mathrm{~nm}$. The rats in the control group received the EB injection only. Data are presented as the mean \pm standard deviation $(n=10)$. $* P<0.05$ versus control; $* * * P<0.00$ I versus control; NS, $P>0.05$ versus control.

Abbreviations: Con, control; EB, Evans blue.

MTX-liposome-coupled microbubbles were administered with ultrasound application, the concentration of MTX in the parietal lobe after treatment increased to $25.3 \pm 2.4 \mu \mathrm{g} / \mathrm{g}$, which was 8.7 -fold greater than that of MTX-liposomecoupled microbubbles alone and 3.6-fold greater than that of MTX alone. In addition, rats treated with MTX-liposomecoupled microbubbles had a significantly higher brain MTX concentration than those treated with unmodified microbubbles and MTX after sonication $(P<0.001$; Figure 7$)$.

\section{Histological analysis}

To evaluate the safety of ultrasound exposure in this study, we examined the hematoxylin-eosin-stained brain tissues after

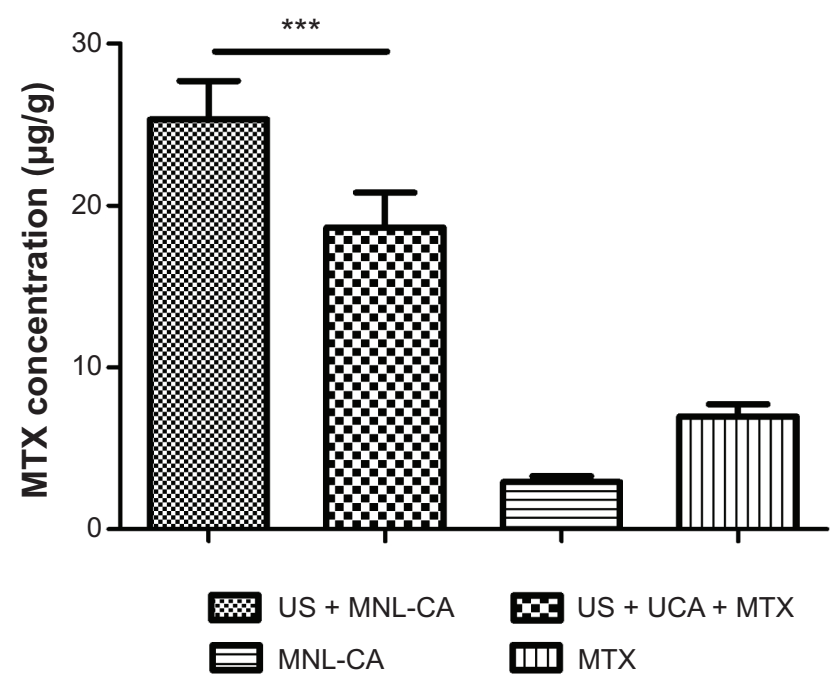

Figure 7 Concentration of MTX in the parietal lobe of the rat brain after treatment with MTX-liposome-coupled microbubbles with or without ultrasound.

Notes: Data are presented as the mean \pm standard deviation $(n=10)$. $* * * P<0.001$. Abbreviations: MTX, methotrexate; US, ultrasound; MNL-CA, MTX-liposomecoupled microbubbles; UCA, unmodified ZHUFIXIAN. the rats were exposed to the optimal ultrasound treatment described above. No tissue or capillary damage was noted in the rats treated with sonication alone (Figure 8) or in combination with MTX-liposome-coupled microbubbles (data not shown). Furthermore, we examined the treatment-induced changes in microvessels in the rat cerebral cortex and hippocampus using lanthanum nitrate, a tight junction marker. TEM images revealed microvessels with intact tight junctions in the control rats and the rats treated with ultrasound only, where the lanthanum tracer was constrained within the microvessels. In the rats treated with MTX-liposome-coupled microbubbles and ultrasound, lanthanum deposition outside of the basement membrane of the vascular wall was observed. In addition, open tight junctions were observed, along with lanthanum deposition, between the nerve fibers (Figure 9).

\section{Discussion}

Optimal treatment of primary CNS lymphoma remains a great medical challenge. Whole-brain radiotherapy is increasingly questioned due to long-term neurotoxicity, especially in elderly patients. In addition, the drugs used to treat non-Hodgkin lymphoma are mostly ineffective, owing to difficulties crossing the BBB. The single most effective therapeutic agent is MTX. Current strategies to achieve adequate MTX delivery to the CNS include the use of highdose MTX, intrathecal administration, and intracarotid infusion with osmotic BBB disruption. ${ }^{26}$ However, each of these strategies has its limitations. High doses of MTX $\left(>3 \mathrm{~g} / \mathrm{m}^{2}\right)$ cause severe systemic toxicity and require intensive inpatient care. Intrathecal administration is highly invasive and may cause infections or hemorrhagic complications. Therefore,

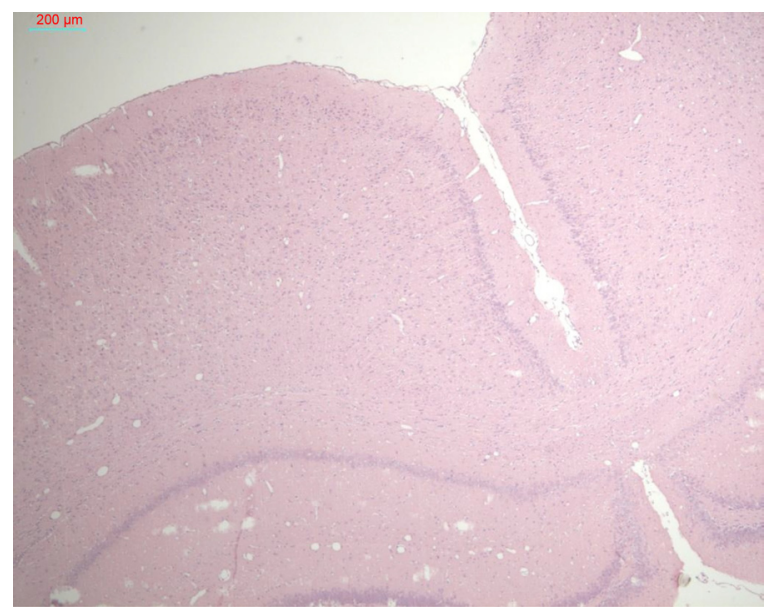

Figure $8 \mathrm{H} \& \mathrm{E}$-stained brain tissue after optimal ultrasound treatment. Notes: Every tenth brain section was stained with H\&E. No tissue or capillary damage was noted in rats treated with sonication. An example section is shown. Bar $200 \mu \mathrm{m}$.

Abbreviation: H\&E, hematoxylin-eosin. 


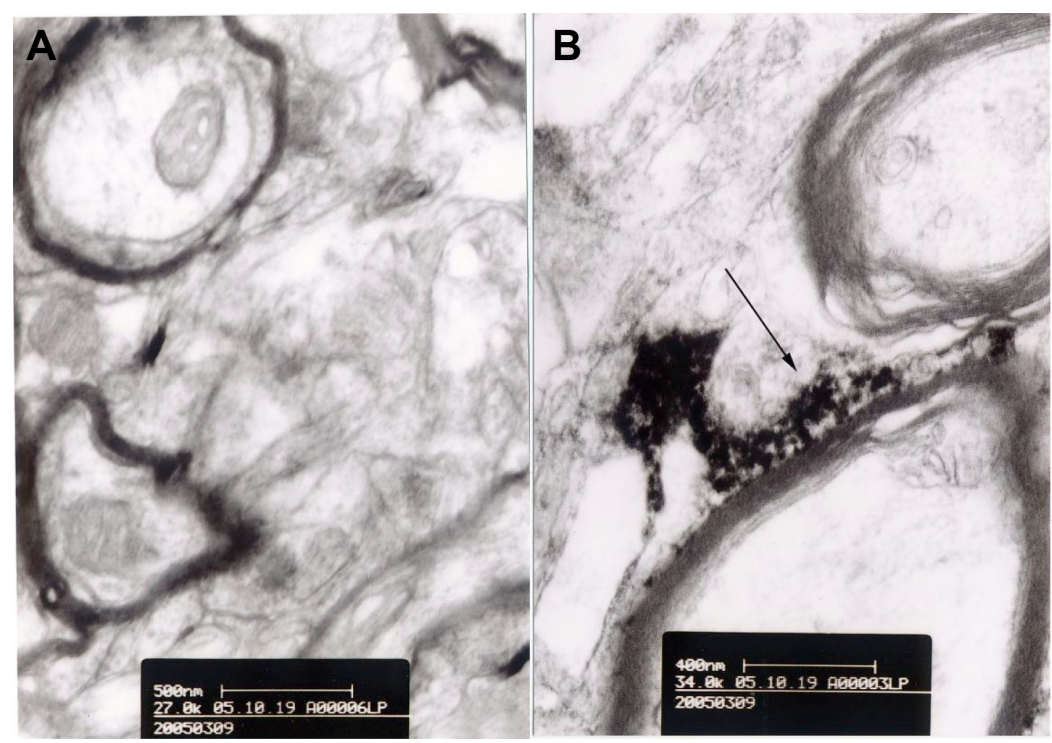

Figure 9 Transmission electron microscopic images of lanthanum nitrate-perfused rat brain.

Notes: (A) Control rats; bar $500 \mathrm{~nm}$. (B) Rats treated with methotrexate-liposome-coupled microbubbles and ultrasound showing lanthanum deposition outside the basement membrane of the vascular wall. The arrow shows lanthanum deposition between the nerve fibers; bar $400 \mathrm{~nm}$.

alternative methods for safe and effective brain MTX delivery are much needed to improve the treatment of this disease.

Since Hynynen et al reported in 2001 that the BBB could be temporarily opened using microbubble contrast agents and ultrasound, this method has been intensively exploited for the noninvasive brain-targeted delivery of CNS drugs. In this study, we investigated the brain-targeted delivery of MTX using MTX-carrying ZHIFUXIAN microbubbles with ultrasound. To overcome the low drug-loading capacity of ZHIFUXIAN, we coupled ZHIFUXIAN microbubbles to MTX-loaded liposomes via biotin-avidin interactions (MTXliposome-coupled microbubbles). These microbubbles had a drug-loading capacity of $8.91 \% \pm 0.86 \%$, which is much higher than that of ZHIFUXIAN and other microbubbles directly loaded with therapeutic/diagnostic agents. ${ }^{27,28}$ Liposome coupling only slightly increased the size of the microbubbles without compromising their acoustic behavior, as they worked as well as ZHIFUXIAN in contrast-enhanced ultrasound imaging (data not shown).

Next, we evaluated the in vitro cytotoxicity of these MTX-liposome-coupled microbubbles. Compared with MTX or MTX-loaded liposomes, these microbubbles showed a reduced cell killing effect against Walker-256 cancer cells if used without sonication. However, they became significantly more toxic than MTX and MTX-loaded liposomes after sonication. Similarly, these microbubbles, if used without sonication, were less potent than MTX and MTX-loaded liposomes in inducing the apoptosis of Walker-256 cells, but became significantly more potent after sonication. Collectively, these results suggest that MTX is encapsulated in the microbubbles under normal conditions and that sonication triggers the release of free MTX and MTX-loaded liposomes from the microbubbles and promotes their cellular uptake via membrane permeation.

We then studied the effects of the MTX-liposome-coupled microbubbles and sonication on BBB permeability in rats, using $\mathrm{EB}$ as a $\mathrm{BBB}$ probe. Similar to previous reports, ${ }^{29}$ the level of BBB opening was dependent upon multiple factors, in the order of ultrasound intensity $>$ duty cycle $>$ duration of sonication $>$ microbubble dose. We found that under optimal treatment conditions $\left(1 \mathrm{mHz}, 2 \mathrm{~W} / \mathrm{cm}^{2}\right.$, duty cycle of $20 \%$, sonication for 5 minutes, and $0.5 \mathrm{~mL} / \mathrm{kg}$ MTXliposome-coupled microbubbles), significant BBB opening could be achieved without noticeable tissue or capillary damage. Under these conditions, EB extravasation was not observed with ultrasound alone or microbubbles alone. We also noted that the location and extent of EB extravasation were correlated with the direction and focal sites of sonication, indicating targeted disruption of the BBB.

To investigate the mechanisms underlying the BBB opening effects of the MTX-liposome-coupled microbubbles and ultrasound, we performed TEM analysis of brain tissues after perfusion with lanthanum nitrate. In rats treated with ultrasound only, lanthanum deposition in the basement membrane of the vascular wall was observed; however, all lanthanum was still constrained within the microvessels, indicating that the tight junctions were intact. After treatment with MTX-liposome-coupled microbubbles and ultrasound, the tight junctions were disrupted, resulting in permeation of lanthanum through the tight junctions and the basement 
membrane into the surrounding tissues. These results indicate that MTX-liposome-coupled microbubbles and sonication increase the BBB permeability by disrupting the endothelial tight junctions of cerebral microvessels.

Finally, we evaluated brain MTX delivery by analyzing the MTX concentration in rat brains using HPLC. The brain MTX concentration after treatment followed the order of MTX-liposome-coupled microbubbles + ultrasound $(25.3 \pm 2.4 \mu \mathrm{g} / \mathrm{g})>$ unmodified ZHIFUXIAN + MTX + ultrasound $(18.6 \pm 2.2 \mu \mathrm{g} / \mathrm{g})>\operatorname{MTX}(6.97 \pm 0.75 \mu \mathrm{g} / \mathrm{g})>\mathrm{MTX}-$ liposome-coupled microbubbles $(2.92 \pm 0.39 \mu \mathrm{g} / \mathrm{g})$. Treatment with MTX-liposome-coupled microbubbles and ultrasound resulted in a significantly higher brain MTX concentration than all other treatments $(P<0.001)$. However, if sonication was not applied, the brain MTX concentration after treatment with MTX-liposome-coupled microbubbles was less than that after treatment with free MTX. These results suggest that the microbubbles release MTX only after sonication, similar to the findings from our in vitro studies.

Acoustically active microbubbles have been actively exploited as drug carriers for the noninvasive and braintargeted delivery of therapeutic agents for CNS diseases. However, their application is limited by their low drug-loading capacity. ${ }^{30}$ In this report, we prepared MTX-liposomecoupled microbubbles with a high drug-loading capacity of $4.91 \pm 0.51 \mathrm{mg} / \mathrm{mL}$. These microbubbles are acoustically active; and their sizes are suitable for intravenous injection. When used with ultrasound, they are cytotoxic against cancer cells in vitro and effectively deliver MTX to the brain via targeted disruption of the BBB in vivo. These microbubbles may hold great promise as new effective therapies for primary CNS lymphoma and other CNS malignancies.

\section{Acknowledgment}

This work was supported by grants from the National Science Foundation of China (30901389 and 30800273).

\section{Disclosure}

The authors report no conflicts of interest in this work.

\section{References}

1. Pardridge WM. The blood-brain barrier: bottleneck in brain drug development. NeuroRx. 2005;2(1):3-14.

2. Buahin KG, Brem H. Interstitial chemotherapy of experimental brain tumors: comparison of intratumoral injection versus polymeric controlled release. J Neurooncol. 1995;26(2):103-110.

3. Guerin C, Olivi A, Weingart JD, Lawson HC, Brem H. Recent advances in brain tumor therapy: local intracerebral drug delivery by polymers. Invest New Drugs. 2004;22(1):27-37.

4. Malerba F, Paoletti F, Capsoni S, Cattaneo A. Intranasal delivery of therapeutic proteins for neurological diseases. Expert Opin Drug Deliv. 2011;8(10):1277-1296.
5. Lochhead JJ, Thorne RG. Intranasal delivery of biologics to the central nervous system. Adv Drug Deliv Rev. 2012;64(7):614-628.

6. Mittal D, Ali A, Md S, Baboota S, Sahni JK, Ali J. Insights into direct nose to brain delivery: current status and future perspective. Drug Deliv. 2014;21(2):75-86.

7. Kumar M, Kakkar V, Mishra AK, Chuttani K, Kaur IP. Intranasal delivery of streptomycin sulfate (STRS) loaded solid lipid nanoparticles to brain and blood. Int J Pharm. 2014;461(1/2):223-233.

8. Oldendorf WH, Hyman S, Braun L, Oldendorf SZ. Blood-brain barrier: penetration of morphine, codeine, heroin, and methadone after carotid injection. Science. 1972;178(4064):984-986.

9. Pardridge WM, Boado RJ. Reengineering biopharmaceuticals for targeted delivery across the blood-brain barrier. Methods Enzymol. 2012; 503:269-292.

10. Rapoport SI. Advances in osmotic opening of the blood-brain barrier to enhance CNS chemotherapy. Expert Opin Investig Drugs. 2001; 10(10):1809-1818.

11. Matsukado K, Sugita M, Black KL. Intracarotid low dose bradykinin infusion selectively increases tumor permeability through activation of bradykinin B2 receptors in malignant gliomas. Brain Res. 1998; 792(1):10-15.

12. Bellavance MA, Blanchette M, Fortin D. Recent advances in blood-brain barrier disruption as a CNS delivery strategy. AAPS J. 2008;10(1):166-177.

13. Mesiwala AH, Farrell L, Wenzel HJ, et al. High-intensity focused ultrasound selectively disrupts the blood-brain barrier in vivo. Ultrasound Med Biol. 2002;28(3):389-400.

14. Hynynen K, McDannold N, Vykhodtseva N, Jolesz FA. Noninvasive MR imaging-guided focal opening of the blood-brain barrier in rabbits. Radiology. 2001;220(3):640-646.

15. Aryal M, Arvanitis CD, Alexander PM, McDannold N. Ultrasoundmediated blood-brain barrier disruption for targeted drug delivery in the central nervous system. Adv Drug Deliv Rev. 2014;72:94-109.

16. Liu HL, Fan CH, Ting CY, Yeh CK. Combining microbubbles and ultrasound for drug delivery to brain tumors: current progress and overview. Theranostics. 2014;4(4):432-444.

17. Tartis MS, McCallan J, Lum AF, et al. Therapeutic effects of paclitaxel-containing ultrasound contrast agents. Ultrasound Med Biol. 2006;32(11):1771-1780.

18. Lentacker I, Geers B, Demeester J, De Smedt SC, Sanders NN. Design and evaluation of doxorubicin-containing microbubbles for ultrasoundtriggered doxorubicin delivery: cytotoxicity and mechanisms involved. Mol Ther. 2010;18(1):101-108.

19. Sirsi SR, Borden MA. Advances in ultrasound mediated gene therapy using microbubble contrast agents. Theranostics. 2012;2(12): $1208-1222$.

20. Tinkov S, Winter G, Coester C, Bekeredjian R. New doxorubicinloaded phospholipid microbubbles for targeted tumor therapy. Part I. Formulation development and in-vitro characterization. J Control Release. 2010;143(1):143-150.

21. Sirsi SR, Fung C, Garg S, Tianning MY, Mountford PA, Borden MA. Lung surfactant microbubbles increase lipophilic drug payload for ultrasound-targeted delivery. Theranostics. 2013;3(6):409-419.

22. Kheirolomoom A, Dayton PA, Lum AF, et al. Acoustically-active microbubbles conjugated to liposomes: characterization of a proposed drug delivery vehicle. J Control Release. 2007;118(3):275-284.

23. Liu P, Wang X, Zhou S, Hua X, Liu Z, Gao Y. Effects of a novel ultrasound contrast agent with long persistence on right ventricular pressure: comparison with SonoVue. Ultrasonics. 2011;51(2):210-214.

24. Gao YH, Tan KB, Liu P. Experimental study on a new long circulation ultrasound contrast agent enhancing heart imaging. Chinese J Ultrasound Med. 2004;20(2):81-84. Chinese.

25. Yang YN, Gao YH, Tan KB, et al. Blood brain barrier permeability of Cisplatin increase induced by transcranial ultrasound and microbubbles in an in vitro model. Chin J Med Imaging Technol. 2009;25(6):938-941.

26. Morris PG, Abrey LE. Therapeutic challenges in primary CNS lymphoma. Lancet Neurol. 2009;8(6):581-592. 
27. Liu H, Wang X, Tan KB, et al. Molecular imaging of vulnerable plaques in rabbits using contrast-enhanced ultrasound targeting to vascular endothelial growth factor receptor-2. J Clin Ultrasound. 2011;39(2):83-90.

28. Yang D, Gao YH, Tan KB, et al. Inhibition of hepatic fibrosis with artificial microRNA using ultrasound and cationic liposome-bearing microbubbles. Gene Ther. 2013;20(12):1140-1148.
29. Wang F, Shi Y, Lu L, et al. Targeted delivery of GDNF through the blood-brain barrier by MRI-guided focused ultrasound. PLoS One. 2012;7(12):e52925.

30. Sirsi SR, Borden MA. State-of-the-art materials for ultrasound-triggered drug delivery. Adv Drug Deliv Rev. 2014;72(1):3-14.

International Journal of Nanomedicine

\section{Publish your work in this journal}

The International Journal of Nanomedicine is an international, peerreviewed journal focusing on the application of nanotechnology in diagnostics, therapeutics, and drug delivery systems throughout the biomedical field. This journal is indexed on PubMed Central, MedLine, CAS, SciSearch $®$, Current Contents $® /$ Clinical Medicine,

\section{Dovepress}

Journal Citation Reports/Science Edition, EMBase, Scopus and the Elsevier Bibliographic databases. The manuscript management system is completely online and includes a very quick and fair peer-review system, which is all easy to use. Visit http://www.dovepress.com/ testimonials.php to read real quotes from published authors.

Submit your manuscript here: http://www.dovepress.com/international-journal-of-nanomedicine-journal 